\title{
Heterogeneidade da Capacidade Dissipativa e do Módulo de Young em Superfícies Poliméricas: Contraste de Fase em AFM com Contato Periódico
}

\author{
Carlos A. R. Costa, Márcia M. Rippel, Fernando Galembeck \\ Instituto de Química, UNICAMP
}

\begin{abstract}
Resumo: O contraste de fase em microscopia de força atômica no modo de contato periódico é determinado pela topografia, viscoelasticidade e pelas propriedades adesivas das amostras. A maximização da quantidade de informação extraída das imagens exige que o procedimento de aquisição seja previamente definido através da escolha adequada do ângulo de fase entre a onda aplicada ao cristal piezoelétrico, que provoca a oscilação da sonda, e a onda gerada pela resposta do fotodetector. O efeito da topografia no contraste de fase é minimizado somente se as duas senóides (oscilação da sonda e resposta do fotodetector) forem ortogonais. Escolhendo $\theta=-90^{\circ}$, os domínios brilhantes na imagem são mais dissipativos que os domínios escuros, porém o oposto é observado usando $\theta=+90^{\circ}$. Esta regra é obtida da análise de operação do instrumento, e é experimentalmente verificada com uma amostra preparada pelo recobrimento parcial de uma superfície de mica com um filme de borracha natural. Três diferentes filmes foram analisados e revelaram diferentes padrões de domínios: domínios duros em uma matriz mole são observados em um látex copolimérico sintético, enquanto que um filme de borracha natural apresenta domínios moles em uma matriz mais dura, e um filme compósito de borracha natural com polifosfato de sódio é formado por domínios duros e moles, interpenetrantes.
\end{abstract}

Palavras-chave: SPM, contraste de fase, viscoelasticidade.

\section{Polymer Surface Viscoelastic Heterogeneity: Phase Contrast Using AFM, in the Periodic Contact Mode}

Abstract: Phase contrast in periodic contact mode-AFM is determined by topographic, viscoelastic as well as adhesive properties of the samples. Image interpretation is not straightforward, unless the image acquisition procedure is previously well defined, making an adequate choice of the phase angle $\theta$ between the wave applied to the driving piezo and the wave from the photodetector output. The effect of topography on phase contrast is minimum only if the two senoidal waves (probe oscillation and phododetector response) are ortogonal. By choosing $\theta=-90^{\circ}$, brighter domains in the image are more dissipative than darker domains, but the opposite is observed using $\theta=+90^{\circ}$. This rule is obtained from a simple analysis of the instrument operation, and it is experimentally verified with a sample prepared by partially coating a mica sheet with a natural rubber film cast from solution. Three different latex films were imaged and revealed different domain patterns: harder domains in a softer matrix are observed in a synthetic copolymer latex, while the natural rubber latex (NRL) has softer domains in a harder continuum, and a NRL-sodium polyphosphate composite film is formed by interpenetrating harder and softer domains.

Keywords: SPM, phase contrast, hard and soft domains.

\section{Introdução}

As superfícies poliméricas apresentam heterogeneidades em escala microscópica, que afetam as suas propriedades de adesão, molhabilidade, abrasão e degradação no meio ambiente. Portanto, a identificação de heterogeneidades e o seu mapeamento têm grande importância científica e tecnológica. Muitas técnicas são utilizadas no estudo de superfícies de polímeros e determinação de suas propriedades, por 
exemplo: ESCA, ângulos de contato, refletânciaFTIR, espectroscopia de frequência-soma, microdureza, resistência DC e impedância AC, entre outras. Entretanto, nenhuma destas técnicas tem resolução espacial em escala nanométrica, no plano da superfície do polímero.

Uma técnica que tem sido utilizada para obter informações sobre superfícies poliméricas com resolução nanométrica é a microscopia de força atômica no modo de contato periódico ${ }^{[1]}$. Esta técnica é baseada em uma sonda de silício que oscila, em sua freqüência natural de ressonância, sobre a superfície de uma amostra tocando-a periodicamente. Durante uma varredura, as variações na interação sonda-amostra, causam mudanças na freqüência, amplitude e fase da vibração da sonda.

Freqüentemente as imagens topográficas são geradas mantendo-se a amplitude da oscilação constante, por meio de uma malha de realimentação que controla a distância entre a sonda e a amostra. Uma segunda imagem pode ser adquirida simultaneamente, cujo contraste é determinado pela defasagem do sinal.

Esta imagem pode revelar vários tipos de heterogeneidades na capacidade de dissipação de energia mecânica da superfície polimérica.

Os principais fatores de dissipação de energia mecânica da sonda e conseqüente alteração de fase são as propriedades viscoelásticas, adesivas e topográficas da região que está sendo examinada. Para se conseguir separar estes vários tipos de contribuições, é necessário realizar um estudo cuidadoso fazendo a sonda varrer a amostra sob várias condições operacionais.

As mudanças de fase da oscilação da sonda e portanto o contraste de fase das imagens pode depender de um grande número de parâmetros, como a amplitude livre de oscilação, a razão entre a amplitude livre e a amplitude mantida durante uma varredura, $o$ ambiente de operação, a geometria da sonda, além de características morfológicas e químicas da superfície $^{[2]}$. A partir da interação destes parâmetros, as imagens de contraste de fase podem ser otimizadas para mapear diferentes características mecânicas da superfície. Segundo Raghvan e colaboradores ${ }^{[3]}$, se a força aplicada pela sonda sobre a superfície for grande, isto é, a razão entre a amplitude de oscilação livre e a amplitude de varredura for mantida em $50 \%$ ou menos, a imagem de contraste de fase é dominada pelas variações nos parâmetros viscoelásticos dos diferentes domínios da superfície. Por outro lado, diminuindo esta força, ou seja, para razões entre a amplitude livre e a amplitude de varredura maior que $50 \%$, predominam as variações na adesão entre a sonda e a superfície.

O efeito da topografia da superfície sobre o contraste das imagens de fase pode ser minimizado através da escolha de uma defasagem de $90^{\circ}$, entre a onda aplicada ao piezo e o sinal do fotodetetor, como está mostrado neste trabalho.

\section{Experimental}

\section{Preparação das amostras}

As amostras examinadas são filmes de látex de borracha natural (NRL) extraído de clones RRIM 600 (Instituto Agronômico de Campinas, SP). Este foi centrifugado a 10krpm ( Sorvall RC 26 Plus, DuPont) por $3 \mathrm{~h}$ a $5{ }^{\circ} \mathrm{C}$, separando a fração sobrenadante que contém as partículas de borracha. Estas foram redispersas em água deionisada obtendo-se um teor de sólidos de $11 \%$. Desta dispersão $5 \mu \mathrm{L}$ foram gotejados sobre mica recém-clivada, secando ao ar e obtendo-se um filme com $400 \mathrm{~nm}$ de espessura média.

Filmes compósitos de borracha natural e polifosfato de sódio (NaPP) foram preparados da seguinte forma: polifosfato de sódio foi adicionado a uma dispersão de látex de borracha natural (razão $\mathrm{NaPP} /$ látex $=0.4$ em massa), sob agitação e temperatura ambiente. Foram gotejados $5 \mu \mathrm{L}$ desta dispersão sobre micra recém-clivada obtendo-se um filme com espessura média de $400 \mathrm{~nm}$.

Outros filmes de borracha natural (NR) foram preparados dissolvendo a borracha seca em tetracloreto de carbono, e gotejando-se a solução sobre mica recém-clivada.

Látex de PSAAM, um copolímero de estireno e acrilamida, foi preparado por polimerização em emulsão, sem surfactante, utilizando persulfato de potássio como iniciador ${ }^{[4]}$, e obtendo-se um teor de sólidos na dispersão de $8,6 \%$.

Filmes deste látex foram preparados gotejando 5 $\mathrm{mL}$ desta dispersão sobre mica, secando ao ar.

\section{Microscopia de varredura por sonda.}

Foi utilizado um microscópio modelo Discoverer TMX 2010 (TopoMetrix) operando no modo de força atômica de contato periódico, sob condições ambientais 
$\left(25^{\circ} \mathrm{C}\right.$ e umidade relativa de $50 \%$ ). As imagens topográficas e de contraste de fase foram obtidas simultaneamente utilizando sondas de silício com raio de afiação de $20 \mathrm{~nm}$, constante de mola de $50 \mathrm{~N} / \mathrm{m}$ e freqüência de ressonância de aproximadamente $100 \mathrm{kHz}$. A velocidade de varredura foi de meia linha por segundo. A razão entre a amplitude de oscilação livre e a amplitude mantida durante uma varredura foi mantida constante em $50 \%$, minimizando os efeitos da adesividade e maximizando os efeitos da viscoelasticidade.

Para caracterizar as respostas mecânicas obtidas dos diferentes domínios foram utilizadas nanoindentações utilizando o mesmo tipo de sonda de silício, porém com constante de mola de $3 \mathrm{~N} / \mathrm{m}$.

\section{Resultados e Discussão}

Inicialmente, é determinado o ângulo de fase entre o sinal elétrico alimentador do piezo que faz oscilar a sonda, e o sinal de corrente obtido pelo fotodetector, que faz a leitura da deflexão do laser pela sonda. Isto é feito durante uma oscilação livre, na freqüência de ressonância. A Figura 1 mostra os pontos do equipamento nos quais se mostra estes sinais.

A seguir, a sonda é aproximada da superfície da amostra até que a amplitude de oscilação seja metade do valor determinado na oscilação livre. Durante uma varredura, estas condições são mantidas constantes, usando-se a malha de realimentação. O sinal da sonda tende a se atrasar quando toca regiões mais macias, mais adesivas ou de topografia descendente (declives) da amostra, e tende a se adiantar em regiões mais duras, não adesivas ou de topografia ascendente (aclives).

$\mathrm{O}$ esquema da Figura 2 ilustra as diferentes pos-

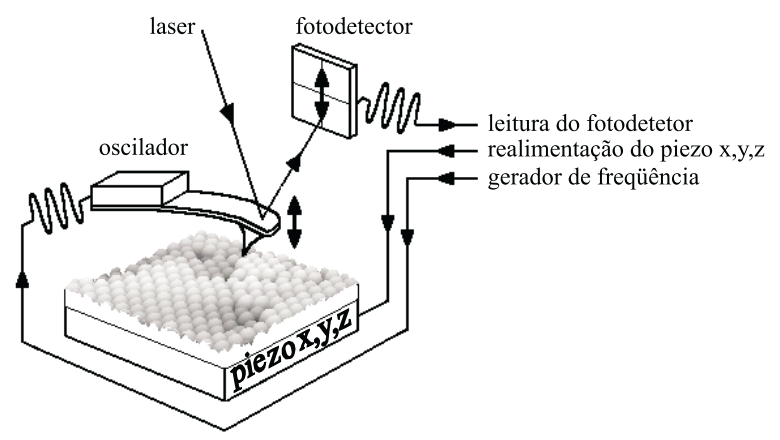

Figura 1. Esquema de operação do microscópio de força atômica com contato intermitente, mostrando duas senóides: uma representa a oscilação do piezo solidário com a sonda, outra representa o sinal do fotodetector.
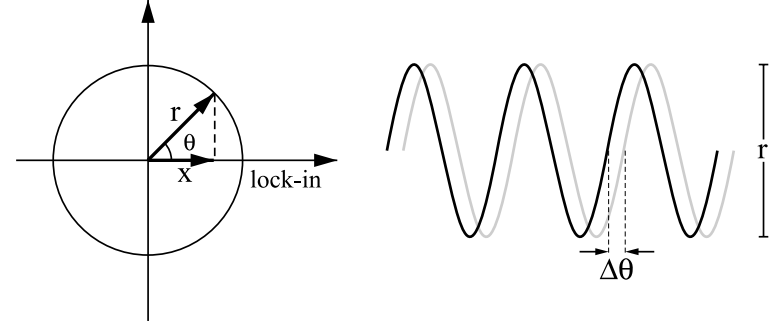

Figura 2. As imagens de contraste de fase são geradas com o sinal (r) detectado no eixo x por um amplificador tipo lock-in. Dependendo do ângulo de fase adotado inicialmente $(\theta)$ entre a oscilação da sonda e a leitura do fotodetector, o sinal x pode aumentar ou diminuir para a mesma variação de fase $(\Delta \theta)$.

sibilidades de aquisição do sinal em função do ângulo de fase.

Variações topográficas provocam alterações na amplitude (r) que tendem a um mínimo com os parâmetros de realimentação devidamente ajustados. Porém, mesmo estas pequenas variações $(\Delta \mathrm{r})$ podem ser interpretadas como variações de fase. Este efeito é detectado em x proporcionalmente ao co-seno do ângulo $\theta$, ou seja, é maximizado em ângulos pequenos e minimizado em ângulo reto.

A Figura 3 mostra imagens de contraste de fase de um filme de NR preparado por secagem de uma solução de NR em tetracloreto de carbono. Para maximizar a contribuição das variações das propriedades viscoelásticas, o sinal (x) foi detectado nos ângulos $\theta$ de $-90^{\circ}$ e $+90^{\circ}$. Também para minimizar os efeitos de $\Delta \mathrm{r}$, o sinal usado na construção da imagem foi a razão entre o sinal (x) e a amplitude (r), ou seja, um sinal dependente apenas das variações do co-seno de $\theta$.

Nestas imagens, a tonalidade mais clara representa a leitura de maior tensão na saída do lock-in. Quando $\theta$ $=-90^{\circ}$, a área recoberta com borracha apresenta-se mais clara que a superfície da mica. Nesta condição, o aumento da tensão de saída do lock-in indica que houve atraso na oscilação da sonda, mas esta permanece constante enquanto a sonda está sobre a borracha. $\mathrm{O}$ atraso é devido à maior capacidade de dissipação viscosa da amostra, com relação à sua capacidade de armazenagem elástica. A diminuição da voltagem sobre a mica indica o adiantamento do sinal, sobre a região mais dura (isto é, a superfície de mica). Para $\theta=+90^{\circ}$, o contraste é invertido: a tonalidade mais clara de uma região da imagem indica tratar-se de uma região dura, e a tonalidade mais escura caracteriza as regiões macias.

As imagens de contraste de fase obtidas para diferentes amostras poliméricas são mostradas na Figura 4. Os filmes possuem partículas de tamanhos e alturas máximas bastante próximas. 


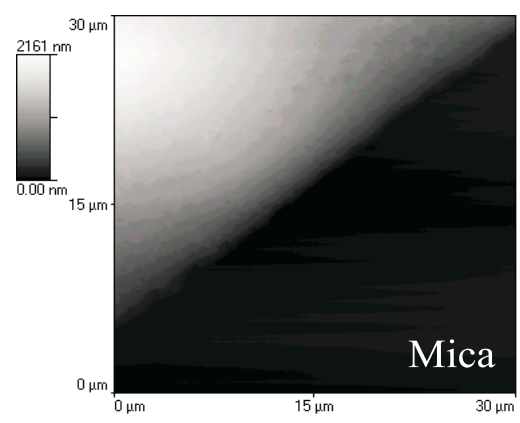

A

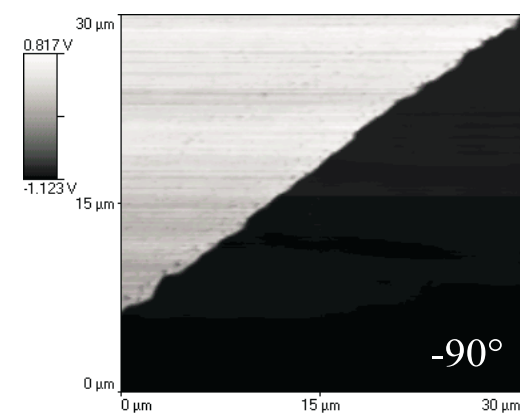

B

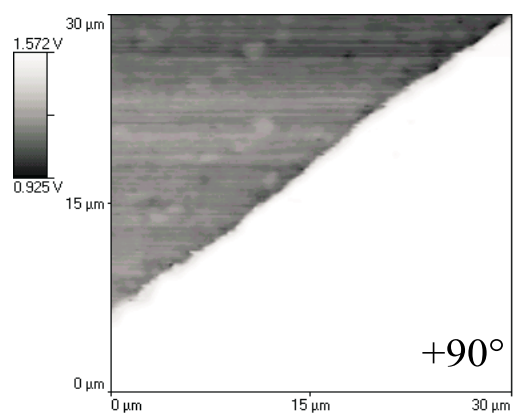

$\mathrm{C}$

Figura 3. A: imagem topográfica da borda de um filme de NR sobre mica; B: imagem de contraste de fase da mesma região adquirida em ângulos $\theta$ de $-90^{\circ}$; C: o mesmo que em $\mathrm{B}$, mas sendo $\theta=+90^{\circ}$. A inversão no ângulo de fase escolhido causa uma inversão no contraste da imagem.

Os contrastes observados nos filmes de PSAAM e de NRL são exatamente opostos: no primeiro, a periferia das partículas é mais macia que o seu centro, enquanto no látex natural o centro é mais macio que a periferia.

No caso do PS-AAM, a maciez periférica é fácil de compreender, considerando os resultados microanalíticos e topoquímicos obtidos anteriormente neste laboratório, para este látex: a casca das partículas é mais rica em acrilamida que o seu interior, e esta é plastificada pela umidade ambiente ${ }^{[5]}$. Por outro lado, o NRL é formado por partículas macias de poliisopreno, rodeadas por cascas ricas em material iônico, que seca formando domínios de maior dureza que a borracha.
Para o compósito de NRL com polifosfato, o contraste obtido é muito acentuado. As imagens mostram que a adição de polifosfato ao látex natural produz domínios interpenetrantes, sendo que estes têm maior tendência à percolação e também uma dureza bastante maior do que a dos domínios mais discretos.

Este resultado mostra a possibilidade de se obter sistemas poliméricos bifásicos formados por domínios muito diferenciados quanto às suas propriedades viscoelásticas, a partir do látex de borracha natural.

A Figura 4 mostra que a resolução lateral alcançada nas imagens de contraste de fase de diferentes materiais é da ordem de $20 \mathrm{~nm}$, permitindo discriminar domínios com diferentes características

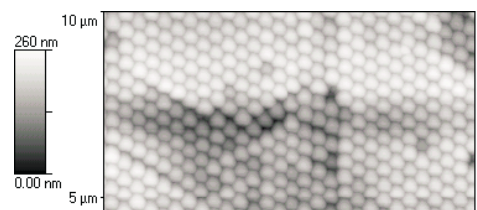

A

A
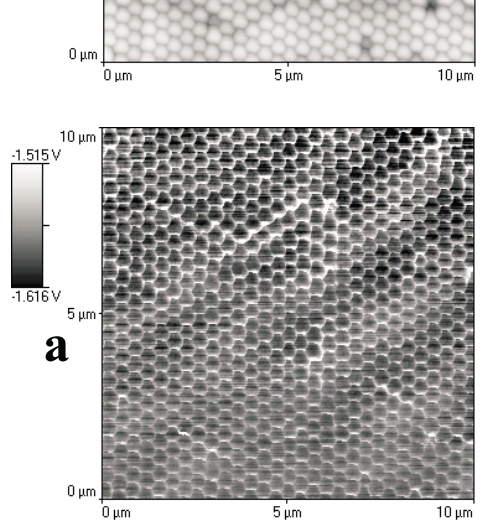
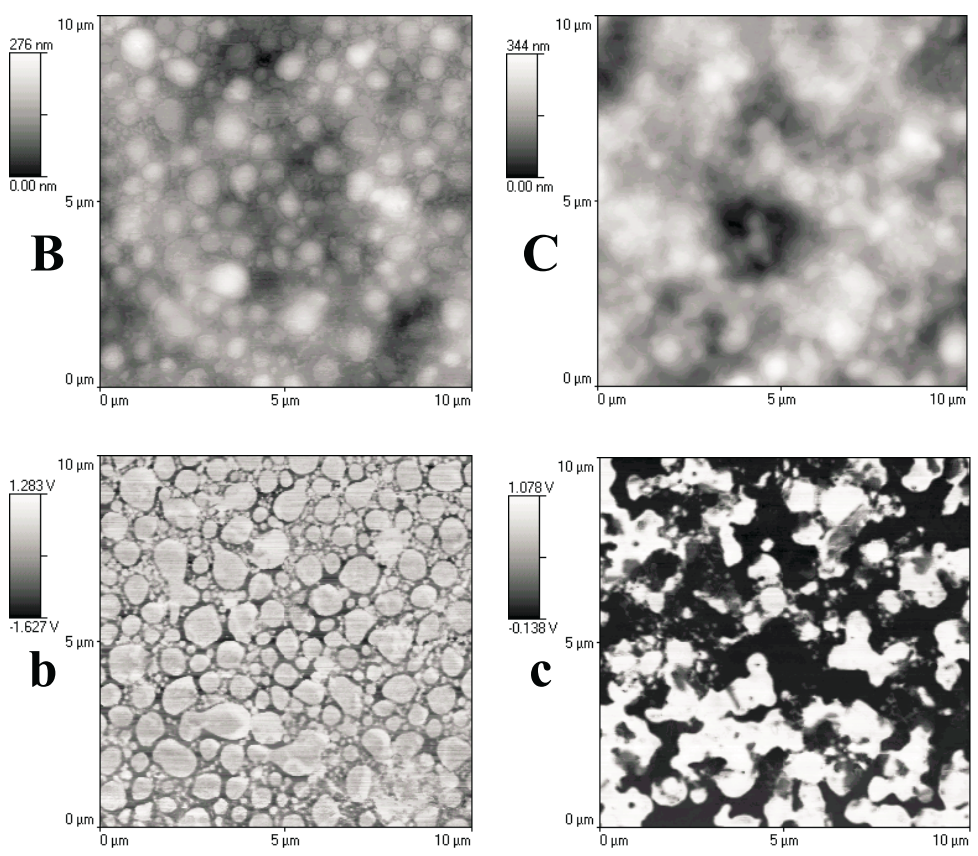

Figura 4. Imagens topográficas de (A) PSAAM, (B) látex natural e (C) látex com polifosfato, com as respectivas imagens de contraste de fase (a, b e c). As imagens de fase foram obtidas ajustando-se $\theta=-90^{\circ}$. 

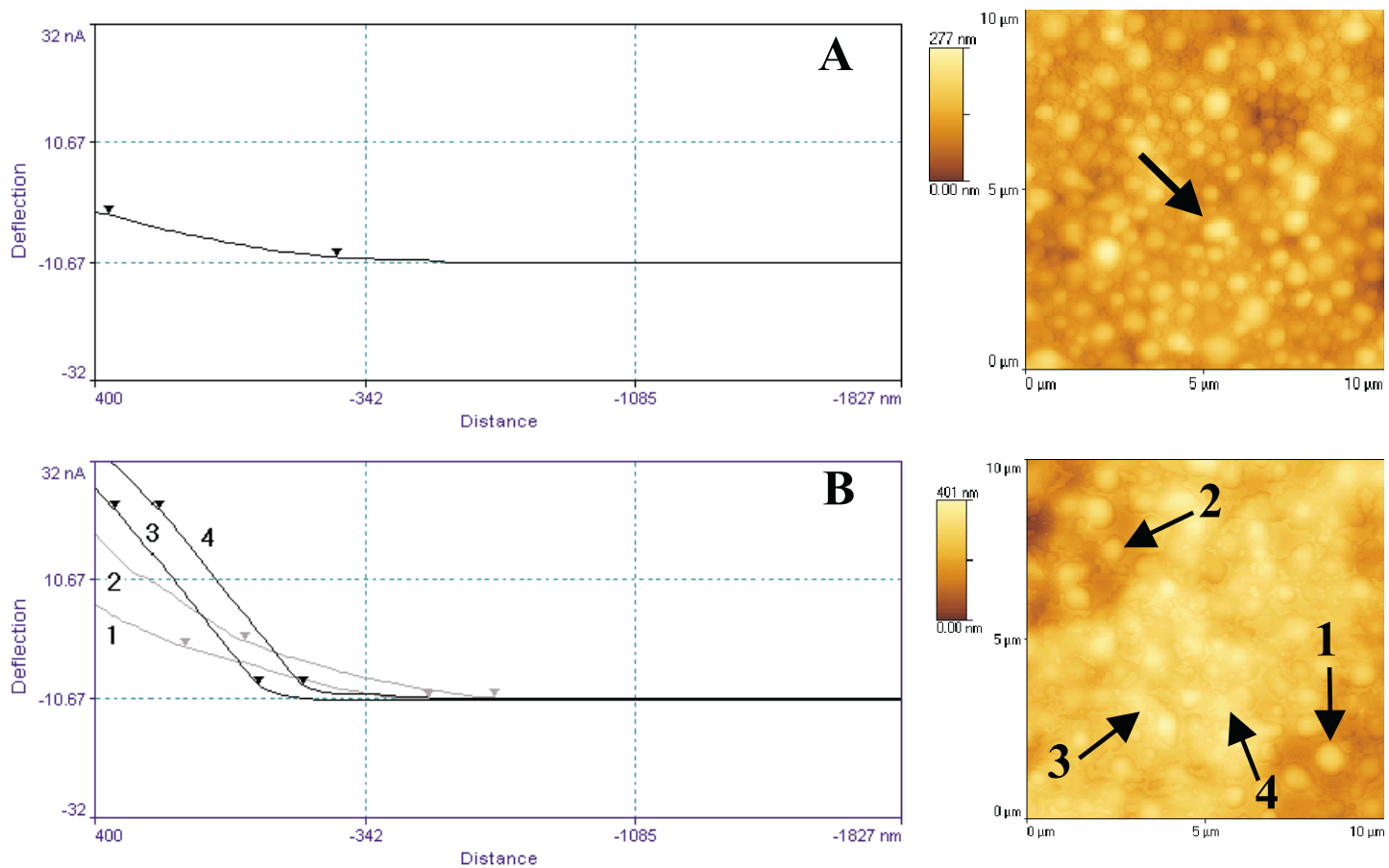

Figura 5. Curvas de força vs. distância (entre a sonda e o filme). A - filme de NRL com 0,458 nN/nm; B- filme de NRL com NaPP, onde os números indicam os vários pontos em que foram executadas as medidas. As inclinações das curvas são: $1=0,624 \mathrm{nN} / \mathrm{nm}, 2=0,665 \mathrm{nN} / \mathrm{nm}, 3=2,949 \mathrm{nN} / \mathrm{nm}$ e $4=2,973 \mathrm{nN} / \mathrm{nm}$

mecânicas e dissipativas com esta resolução lateral.

É possível fazer uma verificação independente de que, nestas condições de aquisição, as regiões mais claras nas imagens de contraste de fase indicam regiões mais macias que as escuras. Medidas de nanoindentação em diferentes regiões dos filmes de NRL e NRL com polifosfato confirmam o contraste viscoelástico observado nas imagens conforme mostram as curvas de força vs. distância na Figura 5, quando a sonda é forçada sobre diferentes pontos do filme.

A inclinação das curvas de força vs. distância, após o contato com a sonda apresentou o valor de $0,458 \mathrm{nN} / \mathrm{nm}$ para o filme de NRL. Com a adição de $\mathrm{NaPP}$ (B), este valor apresentou uma pequena elevação para as partículas de látex agregadas (1 e 2), e valores muito altos da ordem do valor nominal da sonda, sobre os domínios percolantes ricos em polifosfatos (3 e 4). Portanto, estes domínios são tão ou mais duros que a própria sonda, e muito mais duros que a borracha.

\section{Conclusões}

O mecanismo de formação de contraste em microscopia de força atômica de contato periódico se deve às diferentes contribuições locais da superfície da amostra na dissipação da energia mecânica da sonda. $\mathrm{O}$ controle adequado das variáveis de operação torna possível o mapeamento das superfícies poliméricas com domínios de diferentes capacidades de dissipação viscosa e armazenagem elástica, mesmo na presença de variações topográficas de $250 \mathrm{~nm}$ até $2000 \mathrm{~nm}$.

\section{Agradecimentos}

Os autores agradecem à FAPESP, MCT/FINEP/ PRONEX e CNPq pelo apoio financeiro concedido.

\section{Referências Bibliográficas}

1. Magonov, S. N.; Elings, V., Whangbo, M.-H. - Surface Science, 375, 385 (1997).

2. James, P. J.; Antognozzi, M., McMaster, T.J., Newton, J.M., Miles, M.J., Langmuir, 2001, 23, 349.

3. Raghvan, D.; VanLandingham, M., Gu, X., Nguyen, T.Langmuir, 16, 9448 (2000).

4. Suzawa, T.; Fujii, A.; Tamai, H. - J. Colloid Interface Sci., 116, 37 (1987).

5. Teixeira-Neto, E.; Leite, C. A. P.; Braga, M.; Galembeck, F. - J. Colloid Interface Sci., 231, 182 (2000).

Recbido: 10/08/01 Aprovado: 11/06/02 